\title{
Kinematic diffusion approach to describe recharge phenomena in unsaturated fractured chalk
}

\author{
Nicola Pastore ${ }^{1 *}$, Claudia Cherubini ${ }^{2,3}$, Concetta I. Giasi ${ }^{1}$ \\ ${ }^{1}$ DICATECh - Politecnico di Bari, via E. Orabona, 70125 Bari, Italy. \\ ${ }^{2}$ Department of Mechanical, Aerospace \& Civil Engineering - Brunel University London, Uxbridge, United Kingdom. \\ ${ }^{3}$ School of Civil Engineering, University of Queensland, St Lucia, Queensland, Australia. \\ * Corresponding author. E-mail: nicola.pastore@poliba.it
}

\begin{abstract}
When dealing with groundwater resources, a better knowledge of the hydrological processes governing flow in the unsaturated zone would improve the assessment of the natural aquifer recharge and its vulnerability to contamination. In North West Europe groundwater from unconfined chalk aquifers constitutes a major water resource, therefore the need for a good hydrological understanding of the chalk unsaturated zone is essential, as it is the main control for aquifer recharge. In the North Paris Basin, much of the recharge must pass through a regional chalk bed that is composed of a porous matrix with embedded fractures. The case study regards the role of the thick unsaturated zone of the Cretaceous chalk aquifer in Picardy (North of France) that controls the hydraulic response to rainfall. In order to describe the flow rate that reaches the water table, the kinematic diffusion theory has been applied that treats the unsaturated water flow equation as a wave equation composed of diffusive and gravitational components. The kinematic diffusion model has proved to be a convenient method to study groundwater recharge processes in that it was able to provide a satisfactory fitting both for rising and falling periods of water table fluctuation. It has also proved to give an answer to the question whether unsaturated flow can be described using the theory of kinematic waves. The answer to the question depends principally on the status of soil moisture. For higher values of hydraulic Peclet number (increasing saturation), the pressure wave velocities dominate and the preferential flow paths is provided by the shallow fractures in the vadose zone. With decreasing values of hydraulic Peclet number (increasing water tension), rapid wave velocities are mostly due to the diffusion of the flow wave. Diffusive phenomena are provided by matrix and fracture-matrix interaction.

The use of a kinematic wave in this context constitutes a good simplified approach especially in cases when there is a lack of information concerning the hydraulic properties of the fractures/macropores close to saturation.
\end{abstract}

Keywords: Unsaturated; Recharge; Rainfall; Chalk; Kinematic; Diffusive.

\section{INTRODUCTION}

\section{Infiltration in unsaturated chalk}

Chalk can be considered as a dual porosity-dual permeability material where water flow can occur both in the Chalk matrix and through the fractures between the Chalk blocks.

Although it is generally accepted that water movement through the unsaturated chalk is a combination of matrix flow (via the matrix larger pores and microfractures) and fracture (or by-pass) flow, the mechanisms of recharge in fractured chalk are generally, poorly understood, especially as concerns the relative contributions from fractures and matrix. It is not clear what the role is of the fractures in the recharge of the Chalk aquifer, and how solutes are exchanged between the fractures and the matrix (Van den Daele et al., 2007).

In the unsaturated zone, the saturation degree of the chalk determines if the fractures actually play a role in the mobility and retardation of contaminants. Therefore, in unsaturated conditions fractures and matrix may be partially saturated and hydraulic pathways within and between these may be restricted/discontinuous: fractures become 'activated' when the matric potential exceeds a certain threshold. Price et al. (2000) proposed that, in addition to providing a flow pathway, water held by capillary tension on fracture walls could be an important means of storing and supplying water to groundwater.

Therefore, recharge conditions prevailing at the top of the chalk as well as the structural characteristics of the chalk influence the mobility of water and contaminants in the unsaturated chalk. The concomitant effect of these two factors on the mo- bility of water and contaminants in the unsaturated chalk has to be investigated.

The respective contributions of the water transfer through the Chalk matrix or fractures are difficult to determine. The process depends on several parameters: the intrinsic properties of Chalk (porosity, fracture density and aperture, clay fraction, ...), thickness and type of surficial formations (Valdes et al., 2014) and climatic conditions (Ireson and Butler, 2011; Price et al., 2000).

The relative importance of preferential flow in the Chalk and its effect on the recharge process has long been a subject of discussion (Nimmo and Malek-Mohammadi, 2015). As suggested by tracer studies (Van den Daele et al., 2007), fissure flow is primarily responsible for rapid water-table fluctuations. The same study suggests also some pore water exchange from the matrix to fissures.

Brouyère et al. (2004) realized a detailed field and laboratory investigation in the unsaturated zone overlying a chalk aquifer in Belgium to analyse groundwater recharge processes and solute downward migration mechanisms. Results showed that gravitational flows govern groundwater recharge and solute migration mechanisms in the unsaturated zone. In the variably saturated chalk, the migration and retardation of solutes is strongly influenced by recharge conditions. Under intense injection conditions, solutes migrate at high speed along the partially saturated fissures, downward to the saturated zone. At the same time, they are temporarily retarded in the almost immobile water located in the chalk matrix. Under normal recharge conditions, fissures are inactive and solutes migrate 
slowly through the chalk matrix. Moreover, he concluded that the vulnerability of the chalk to contamination is strongly dependent on the existence or not of a protective cover. In the presence of a thick covering layer, the water recharge rate applied at the top of the chalk is strongly attenuated. Fissures remain inactive and the downward migration of water and contaminants across the matrix is very slow. This enhances the possibilities for attenuation mechanisms to reduce contamination levels during the migration across the unsaturated chalk. If the chalk is outcropping or just covered by more pervious materials, under intense recharge conditions, it is likely that fissures would become frequently saturated, driving contaminants at high speed downward to the aquifer and reducing the capacity for natural attenuation in the unsaturated zone. The chalk would thus be more vulnerable in this situation.

In Picardy (North of France), Barhoum et al. (2014) carried out investigations in the underground chalk quarry of Saint Martin le Noeud (Beauvais, France) partially flooded and located at the top of a piezometric dome. The quarry represents an exceptional pilot site because it allows to complete the water balance from the surface, from the soil - unsaturated zone to the saturated zone; this setting is extremely rare in hydrogeology to characterize the input and output of the unsaturated zone. The study demonstrated strong relationships between the quality of groundwater and the characteristics of the superficial formations above the Chalk, the cultural practices and the thickness of the unsaturated zone (Barhoum et al., 2014).

Foster (1975) argued that tritium concentration recorded in pores might occur as a result of diffusion from fissures to matrix. This could happen where the water movement was retarded by low hydraulic conductivity conditions such as smaller fissure apertures. Downing et al. (1979) recorded strong evidence for substantial fissure flow in the Chalk from observations of bacterial contamination after heavy rainfall events. Reeves (1979) studied the literature of field observations in the Chalk and suggested that the major part of the groundwater might be transported through microfissures (with openings of $20 \mu \mathrm{m}$ or less) and very large pores rather than matrix pores.

Price et al. (2000) believed that micro-concavities - referred to as "irregularities" - on the surface of chalk blocks must be responsible for much of the water storage in block samples as the pores would not drain under such low matric potentials. Thinking of fissures in chalk as the gaps between chalk blocks, chalk block surfaces are in fact fissure faces, and the water runs on block surfaces can be considered "fissure-face flow". The data suggest that flow on the outer surfaces of the blocks, analogous to fissure-face flow, is responsible for the major drainage at this range of pore pressure.

\section{Conceptual models of infiltration in unsaturated chalk}

Different types of conceptual models have been formulated to explain infiltration and recharge processes in unsaturated fractured rock.

Traditional conceptual models for flow and transport in unsaturated fractured rock require high fracture saturations in order to permit flow through fractures. The first conceptual model uses the concept of preferential flow, wherein rapid subsurface flow is caused by high fluid velocities in macropores such as fractures (White, 1985). Rapid responses are attributed to water movement through fractures that become active flow conduits, whereas an attenuated response is attributed to matrix flow at larger matric tensions. The preferential flow formulation consists in an aperture-based model of water distribution in unsaturated fractures that depicts a cross section through a variable aperture fracture with water occurring at isolated locations corresponding to contact points or aperture minima. In this conceptualization based strictly on aperture distributions, microscale regions along the fracture plane or local segments within a fracture are either fully saturated (hydraulically conductive) or fully desaturated (non conductive) (Tokunaga and Wan, 1997) such that continuous saturated pathways within fractures are required to sustain fast flow. Field observations at multiple sites are inconsistent with the preferential flow hypothesis, in that water contents are generally too dry to support macropore saturation. Much of the previously mentioned evidence of fast flow in fractures as well as macropores lacks direct evidence of continuous, saturated pathways (Tokunaga and Wan, 1997). Many observations of irregular and fast - developing wetting patterns, suggest that preferential flow commences before macropores are filled (Nimmo and Mitchell, 2013). However, experimental studies have shown that flow proceeds along localized preferential flow paths (e.g., Dahan et al., 1999; Nicholl et al.,1994; Su et al., 1999). For example, an infiltration experiment on a single, in situ, unsaturated fracture in the Negev Chalk, Israel, showed that $70 \%$ of water flux was transmitted through less than $20 \%$ of the studied fracture openings (Dahan et al., 1999). To account for these inconsistencies, hypotheses related to fluid films on fracture surfaces have been proposed (Tokunaga and Wan, 1997). Film flow along fracture surfaces has been recently shown to depend on surface roughness and the matric (capillary) potential (Tokunaga and Wan, 1997). Water film thickness can build up along fracture surfaces when the matric potential is high enough to sustain effectively saturated conditions in the immediately underlying matrix. As matric potentials increase (towards zero), water film thicknesses expand along fracture surfaces by first filling finer scale roughness features, and progressively filling coarser roughness features. The transmissivity (volumetric flux per unit length transverse to flow, under a hydraulic head gradient of unity) of such films increases with film thickness, and supports fast gravity-driven flow. Fast flow of water can therefore occur along partially saturated fractures. Besides rapidity of transport, another major consequence of preferential flow is that the patterns of distribution of water and other transported substances can be irregular; as described by Šimůnek et al. (2003), preferential flow "results in irregular wetting of the soil profile as a direct consequence of water moving faster in certain parts of the soil profile than in others". This phenomenon greatly increases the difficulty of prediction because traditional unsaturated flow models fail to model irregular development of water distribution (Nimmo and Mitchell, 2013).

Recently, John Nimmo has developed the 'Source Responsive Model (SRM)' (Nimmo, 2010), that considers the unsaturated medium as a dual domain of slow matrix flow and rapid preferential flow, the latter causing the patterns of wetting to develop in ways that the traditional theory does not predict. In the SRM, a diffuse domain represents flow within soil matrix material following traditional formulations, and a source responsive domain represents preferential flow conceptualized as laminar flow in free-surface films along the walls of pores. The term source - responsive is used to mean that flow responds sensitively to changing conditions at the source of water input (e.g., rainfall, irrigation, or ponded infiltration). The key feature for source responsive flow is that flowing liquid incompletely fills the space within the macropore and therefore is not fully constrained by capillary forces and solid walls. The flow conditions are little - affected by air - water pressure differences but are sensitive to whether or not the macropore is sup- 
plied with flowing water. Other consequences include that effective macropore aperture has little influence on either flow capacity or speed, and there is no significant effect of buoyancy to inhibit gravitational flow. The SRM approach accounts also for the capability of fractures to store the percolating water partially on their facial micro - concavities that creates conditions suitable for the water and contaminants to get exchanged between the two domains (Nimmo and Malek-Mohammadi, 2015; Price et al., 2000).

An alternative conceptual model uses kinematic theory to predict the pressure wave velocity, or celerity, of unsaturated media (Rasmussen et al., 2000). This formulation assumes that rapid hydraulic responses occur due to semi - compressible flow within the rock matrix. The predicted ratio of the wave velocity to the fluid velocity, or kinematic ratio, is consistent with field observations in unsaturated, fractured rock (Rasmussen, 2001).

Rasmussen et al. (2000) observed irrigation - induced pressure wave velocities that were 1000 faster than tracer velocities in an intact saprolite column. Observed pressure wave velocities were substantially faster than those predicted based on kinematic theory alone. They derived instead a kinematic form of Richards' equation that is mathematically identical to the advection - diffusion equation for a Dirac (spike) input that provides an excellent fit of the observed hydraulic response. This result indicates that rapid pressure head responses were caused by large hydraulic diffusivities. Rapid hydraulic responses arise from the translation and dispersion of a pressure, or energy, wave. This concern is particularly acute when laboratory or field tests confuse the Richards' equation response with either the darcian flux, fluid velocity, or kinematic response. They concluded that pressure pulse velocity monitoring may be an additional tool for estimating unsaturated hydraulic properties in low permeability media.

The present study analyses the recharge in the Cretaceous fractured chalk aquifer in Picardy (North of France) using the kinematic diffusion theory to predict water table fluctuation in response to rainfall. Kinematic diffusion models are useful to predict preferential fluxes and dynamic conditions. The presented approach conceptualizes the unsaturated flow as a combination of 1) diffusive flow that refers to the idealized portion of the pore space of the medium within which the flow rate is driven essentially by local gradient of potential; 2) preferential flow by which water moves across macroscopic distances through conduits of macropore length. A hydraulic form of the advection-diffusion equation based on Richards' equation is proposed where the flow rate replaces the solute concentration, the hydraulic diffusion coefficient replaces the solute diffusion coefficient and the celerity replaces the tracer velocity. The celerity, defined as the derivative of the Darcy flux with respect to the water content, is used in this approach to describe the velocity of movement of a flow rate perturbation through the unsaturated zone, where small changes in fluid saturation within soil matrix pores and fractures govern flow waves propagation.

The objectives of this paper are to introduce a simplified approach to describe recharge phenomena, defining the relative significance of capillary and gravity forces in the process of infiltration in the unsaturated zone, applicable in cases where there is a lack of information concerning the hydraulic properties of the fractures/macropores close to saturation.

\section{Hydrogeological characteristics of the study area}

The study area is in Beauvais, located in the region of Picardy and in the department of Oise, about $80 \mathrm{~km}$ north of Paris. The study area covers $33.31 \mathrm{~km}^{2}$, and is bounded by the city of Fouquenies, Troissereux, Therdonne, Goincourt, Tillé and Mount Saint-Adrien. The area is characterized by numerous rivers that are located in the valley of Therain, the main one is the Oise River, the largest tributary of the Seine River $\left(65,000 \mathrm{~km}^{2}\right)$. In terms of hydrogeology, the Oise river network is connected with two main geological formations: Eocene sands and limestones, and Cretaceous chalk.

The average altitude of the study area is $100 \mathrm{~m}$ above sea level. In Beauvais, the climate is temperate and there is a significant rainfall. The average annual temperature is $10.2^{\circ} \mathrm{C}$ and the average annual rainfall is $669.4 \mathrm{~mm}$ (values measured during the period 1981-2010 provided by Météo France).

The area is characterized by a very productive single aquifer system, recharged by rainfall, where groundwater flows through fractured chalk. The groundwater of the chalk aquifer is extracted for drinking water supply, agriculture and industry.

The chalk aquifer of Beauvais is composed essentially of Cenomanian units about $200 \mathrm{~m}$ thick, which rest on the Albian Gault Clays, an impermeable formation that constitutes the hydrogeological substratum of the chalk aquifer (Roux and Tirat, 1967). The surficial layer is characterized by a clay-withflints cover whose thickness is variable $(0.5-2 \mathrm{~m}, 2-8 \mathrm{~m})$ according to the area (Scheurer, 2000).

The Cenomanian chalk aquifer is characterized by a general groundwater flow towards the south and, more locally, towards the main drains of the Oise river valley (Zouhri and Lutz, 2010). The chalk area is divided in two sectors that are characterized by different topographic and geological features. The western sector consists of a recent alluvial plain limited to the west by clay-with-flints, a relatively impermeable formation (Zghibi et al., 2015). Nevertheless, being the saturated zone in the Chalk aquifer lower than the base of this overlying formation, the former is unconfined.

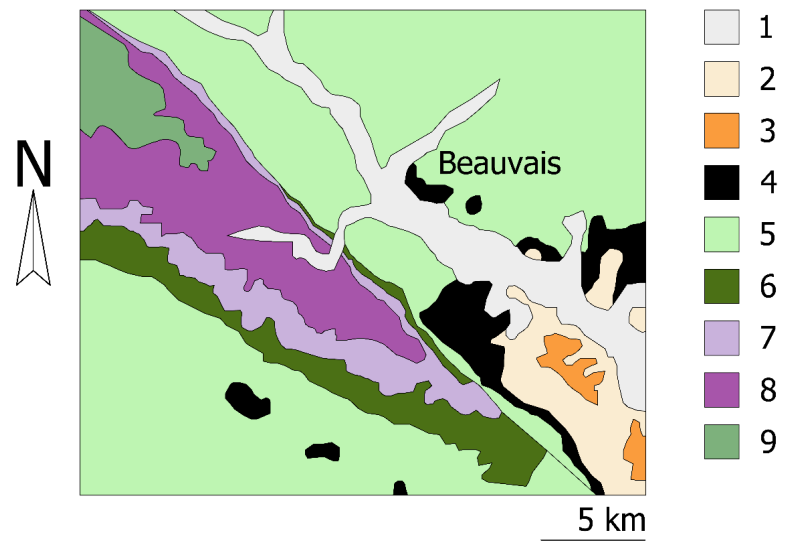

Fig. 1. Geological map of Beauvais area. (modified from Zouhri and Lutz, 2010). 1 Alluvions; 2 Upper Eocene; 3 CuisianSparnacian (medium sand and clay); 4 Thanetian (fine sand); 5 Senonian-Upper Turonian (Chalk with flint); 6 Cenomanian (glauconit chalk); 7 Albian (Clay of Gault (green sand)); 8 Neocomian (sand and clay); 9 Upper Jurassic (alternation of limestone and clay).

The eastern sector is characterized by sequence of limestone and clay also by glauconite chalk separated from the eastern plain of Beauvais by a Bray fault, beyond which the 
Table 1. Geological log of Beauvais site.

\begin{tabular}{ll}
\hline Depth $(\mathrm{m})$ & Geology \\
\hline $0-1.20$ & Filling material \\
$1.20-1.80$ & Dark grey clay \\
$1.80-2.80$ & Black peat \\
$2.80-8.10$ & Coarse siliceous gravel and sands \\
$8.10-10.00$ & Tender pasty chalk \\
$10.00-18.00$ & White chalk with flints, tender, fissured \\
$18.00-20.00$ & Indurated chalk \\
$20.00-22.00$ & More tender chalk \\
\hline
\end{tabular}

aquifer becomes confined (Crampon et al., 1993). Figure 1 shows the geological setting of the study area.

In the unconfined zone, the depth to the water table varies between 50 and $60 \mathrm{~m}$ in the plains and less than $20-30 \mathrm{~m}$ in the humid valleys (Zouhri and Lutz, 2010).

Groundwater flow takes place mostly in the fractured chalk aquifer, characterized by a quite homogeneous hydraulic gradient that varies from 10 to $12 \%$ (Zouhri and Lutz, 2010). Its transmissivity varies with values around $10^{-4} \mathrm{~m}^{2} / \mathrm{s}$ at the centre of the plateau, $10^{-3} \mathrm{~m}^{2} / \mathrm{s}$ in dry valleys and $10^{-1} \mathrm{~m}^{2} / \mathrm{s}$ in humid valleys (Zghibi et al., 2015). The specific yield determined from well test analysis carried out in the area is 0.04 .

The present study is focused on data coming from the French Portail national d'Accès aux Données sur les Eaux Souterraines (ADES) (http://www.ades.eaufrance.fr/) and the French Infoclimat meteorological service (www.infoclimat.fr) concerning daily observations of groundwater level and precipitation for a five-year period (2007-2013). The French observatory ADES has a network of piezometers in whole France and especially 39 in the Oise Department around the Beauvais area, which have been daily monitored since 1974 .

Figure 2 shows a map of the 39 piezometers in the Oise department and the location area of the selected piezometer (the only one in Beauvais area), the meteorological station of Tillé airport and the geological log reported in the Table 1.

Data about precipitations are available in Tillé airport station (north of Beauvais). The mm of precipitation and the rainy days per season during the selected period have been tabulated (Table 2 and Table 3 ).

Table 2. Cumulative precipitation per season for the period 20072013.

\begin{tabular}{cccccc}
\hline & \multicolumn{5}{c}{ Cumulative precipitation (mm) } \\
Year & Winter & Spring & Summer & Autumn & Annual \\
\hline 2007 & & 121.8 & 277.5 & 137.4 & 696.5 \\
2008 & 104.1 & 241.4 & 140.4 & 154.8 & 618.7 \\
2009 & 121.8 & 128 & 125.4 & 160.8 & 560.4 \\
2010 & 182.6 & 103.6 & 154.1 & 152.6 & 561.3 \\
2011 & 88.7 & 19 & 210.4 & 123.1 & 562.1 \\
2012 & 196.8 & 132.4 & 155.2 & 166.4 & 613.1 \\
2013 & 152.4 & 123.3 & 159.4 & 166.8 & 708.6 \\
\hline
\end{tabular}

Table 3. Rainy days per season for the period 2007-2013.

\begin{tabular}{cccccc}
\hline Year & Winter & Spring & $\begin{array}{c}\text { Rainy days (d) } \\
\text { Summer }\end{array}$ & Autumn & Year \\
\hline 2007 & & 48 & 58 & 54 & 214 \\
2008 & 48 & 60 & 45 & 50 & 202 \\
2009 & 39 & 31 & 32 & 51 & 158 \\
2010 & 50 & 32 & 35 & 62 & 172 \\
2011 & 44 & 14 & 58 & 61 & 191 \\
2012 & 54 & 44 & 49 & 49 & 193 \\
2013 & 48 & 44 & 36 & 63 & 231 \\
\hline
\end{tabular}

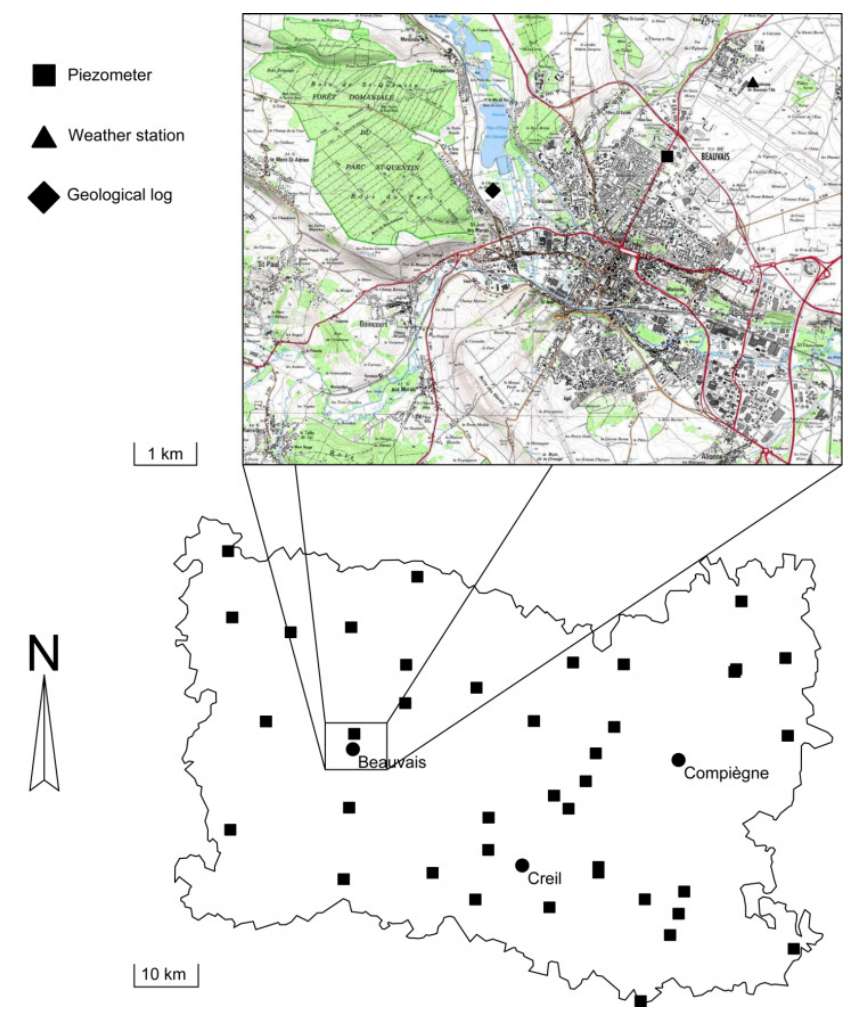

Fig. 2. Location of piezometers in the Oise district from ADES database and location of the geological $\log$, the meteorological station and the selected piezometer (bss code 01024X0058S1) in the Beauvais area.

\section{MATERIALS AND METHODS Kinematic wave theory}

Kinematics is defined as the study of motion excluding the influence of mass and force.

Lighthill and Whitham (1955) developed the kinematic wave theory to describe flood movement in long rivers. The kinematic wave model neglects inertia and pressure gradient terms in the equation of motion and results in a purely convective wave.

Thus, in a kinematic wave the gravity and frictional terms are balanced, so the flow does not accelerate appreciably. For these kinds of waves, the energy grade line is parallel to the channel bottom.

Since its development, this method has gained wide acceptance worldwide and has found widespread application in environmental and water sciences, including watershed runoff modeling, flood routing in rivers and channels, unsaturated flow, macropore flow, flow in furrows and borders, river hydraulics, movement of glaciers, erosion and sediment transport, sedimentation, solute transport, microbial transport and chromatographic transport (Singh, 2002).

Sisson et al. (1980) applied the kinematic wave approach to internal drainage but Smith (1983) was the first to develop a complete kinematic wave model for soil moisture movement and Beven (1982) used it to describe two-dimensional flow in a sloping soil. Charbeneau (1984) extended Smith's work to solute transport and Charbeneau et al. (1989) to multiphase solute transport.

Germann and Beven $(1985,1986)$ extended the application of the theory to infiltration and drainage into and from soil macropores. Yamada and Kobayashi (1988) carried out field tests with tracers to analyse vertical infiltration of soil moisture which they observed having kinematic wave characteristics. 
More recently, Van den Daele et al. (2007) used a modelling package called MACRO (Larsbo et al., 2005) which uses a modified kinematic wave equation to describe flow in fractures under the assumption that they are analogous to soil macropores and Richards' equation for flow in micropores (chalk matrix). The use of the kinematic wave approach implies the assumption that capillary effects in the fracture domain are negligible. The model was unable to simulate the observed matric potential data in the Fleam Dyke lysimeter in Cambridgeshire although it showed good correspondence with the observed water content and drainage flux data (Van den Daele et al., 2007).

\section{Approach: the kinematic diffusion model}

Fluid flow through unsaturated media can be described applying the mass and momentum conservation. Assuming that the density of the fluid is constant and the one dimensional flow in the $\mathrm{z}$ - direction the compressible Euler equations (Yang, 2000) can be written in simplified form as:

$$
C(h) \frac{\partial h}{\partial t}+\frac{\partial q}{\partial z}=0
$$

$$
\frac{1}{g} \frac{\partial q}{\partial t}+\frac{q}{g} \frac{\partial q}{\partial z}+\frac{\partial h}{\partial z}+\frac{q}{K(h)}=0
$$

where $t(\mathrm{~T})$ is the time, $h(\mathrm{~L})$ is the hydraulic head of the unsaturated zone defined as sum of the pressure head and the elevation, $q\left(\mathrm{LT}^{-1}\right)$ is the specific flow rate, $C(h)\left(\mathrm{L}^{-1}\right)$ is the nonlinear capacitive term, $g\left(\mathrm{LT}^{-2}\right)$ is the gravitational acceleration, $K(h)\left(\mathrm{LT}^{-1}\right)$ is the nonlinear equivalent conductivity.

The first two terms of the momentum conservation equation are the inertia terms which can be neglected for the scale of investigation.

Copying the approach used in surface water hydrology to predict flood wave velocity, differentiating Equation (1) with respect to $z$ and Equation (2) with respect to $t$ :

$C \frac{\partial^{2} h}{\partial z \partial t}+\frac{\partial^{2} q}{\partial z^{2}}=0 \Rightarrow \frac{\partial^{2} h}{\partial z \partial t}=-\frac{1}{C} \frac{\partial^{2} q}{\partial z^{2}}$

$\frac{\partial^{2} h}{\partial z \partial t}+\frac{1}{K} \frac{\partial q}{\partial t}-q \frac{1}{K^{2}} \frac{\partial K}{\partial t}=0$

Using the chain rule the time derivative of $K$ becomes:

$\frac{\partial K}{\partial t}=\frac{\partial K}{\partial h}\left(-\frac{1}{C} \frac{\partial q}{\partial z}\right)$

Finally, the parabolic partial differential convection - diffusion equation with dependent variable $q$ can be obtained substituting Equation (3) and Equation (5) into Equation (4):

$$
\frac{\partial q}{\partial t}+\frac{q}{C K} \frac{\partial K}{\partial h} \frac{\partial q}{\partial z}-\frac{K}{C} \frac{\partial^{2} q}{\partial z^{2}}=0
$$

For conditions of steady, vertical, gravity-driven flow equation through the unsaturated zone (where $z$ is taken in the downward direction for convenience) a unit hydraulic gradient, $\nabla h=[0,0,-1]$, is conventionally specified (Sisson et al., 1980). Therefore, assuming that $(\partial h / \partial z=1)$ (Rasmussen at al., 2000) Equation (6) can be written as:

$$
\frac{\partial q}{\partial t}+\frac{1}{C} \frac{\partial K}{\partial h} \frac{\partial q}{\partial z}-\frac{K}{C} \frac{\partial^{2} q}{\partial z^{2}}=0
$$

Equation (7) is mathematically identical to the advection diffusion equation used in solute transport. In this case, the flow rate replaces the solute concentration, the hydraulic diffusion coefficient replaces the solute diffusion coefficient and the celerity replaces the tracer velocity.

The specific flow rate infiltrating through the unsaturated medium undergoes convection and diffusion transport phenomena. It is convected with a kinematic velocity or wave velocity or celerity $c_{\omega}\left(\mathrm{LT}^{-1}\right)$ :

$$
c_{\omega}=\frac{1}{C} \frac{\partial K}{\partial h}
$$

and diffused with a hydraulic diffusivity $D_{\omega}\left(\mathrm{LT}^{-2}\right)$ :

$$
D_{\omega}=\frac{K}{C}
$$

The celerity is used to predict the velocity of movement of a flow rate perturbation through the medium. Unlike saturated media where flow waves propagate due to the compressibility of the fluid, flow waves propagate through unsaturated media as a result of small changes in fluid saturation within soil matrix pores and fractures.

The solution of the advection-diffusion equation for a Dirac input $q(0, t)=q_{0} \delta(t)$ along a semi-infinite boundary (Crank, 1956) is:

$q(z, t)=\frac{q_{0}}{\sqrt{4 \pi D_{\omega} t^{3}}} z \exp \left[\frac{-\left(z-c_{\omega} t\right)^{2}}{4 D_{\omega} t}\right]$

where $q_{0}\left(\mathrm{LT}^{-1}\right)$ is the hydraulic condition at the upper boundary, and $\delta(t)$ is the Dirac function. This formulation assumes that kinematic velocity and hydraulic diffusivity are constant in space and time.

The specific flow rate that reaches the water table can be determined by the convolution product between $q(L, t)$ with $q_{0}$ equal to the unit and the effective precipitation function of a precipitation event $P_{\text {eff }}(t)$. L (L) represents the distance between the soil surface and the water table that changes in time. In order to overcome this issue, the normalized celerity $\left(c_{\omega}^{*}=c_{\omega} / L\right)\left(\mathrm{T}^{-1}\right)$ and the normalized hydraulic diffusivity $\left(D_{\omega}^{*}=D_{\omega} / L^{2}\right)\left(\mathrm{T}^{-1}\right)$ have been introduced.

Then $q(L, t)$ can be expressed as:

$q(L, t)=P_{e f f} * \operatorname{urf}(t)$

where $\operatorname{urf}(t)$ is the unit response function $\left(q_{0}=1\right)$ that assumes the following expression:

$u r f(t)=\frac{1}{\sqrt{4 \pi D_{\omega}^{*} t^{3}}} \exp \left[\frac{-\left(1-c_{\omega}^{*} t\right)^{2}}{4 D_{\omega}^{*} t}\right]$

The unit response function can be characterized using the time moments. 
The mean residence time $t_{m}$ and the degree of spread relative to $t_{\mathrm{m}}$ or the time variance $\sigma^{2}$ represented by the second moment are given by (Brutsaert, 2005):

$$
\begin{aligned}
t_{m} & =\frac{\int_{0}^{\infty} t \cdot u r f(t) d t}{\int_{0}^{\infty} u r f(t) d t} \\
\sigma^{2} & =\frac{\int_{0}^{\infty}\left(t-t_{m}\right)^{2} u r f(t) d t}{\int_{0}^{\infty} u r f(t) d t}
\end{aligned}
$$

The time of the specific flow rate peak in groundwater $t_{p}$ (time of peak) can be determined differentiating $q$ in Equation (10) with respect to $t$ (Yang and Endreny, 2013):

$$
\begin{aligned}
& t_{p}=\frac{1}{2 c_{\varpi}^{2}}\left(-6 D_{\bar{\sigma}}+6 D_{\bar{\sigma}} \sqrt{1+\frac{z^{2} c_{\varpi}^{2}}{9 D_{\varpi}^{2}}}\right) \Rightarrow \\
& t_{p}=\frac{1}{2 c_{\bar{\sigma}}^{* 2}}\left(-6 D_{\bar{\sigma}}^{*}+6 D_{\bar{\sigma}}^{*} \sqrt{1+\frac{z^{2} c_{\varpi}^{* 2}}{9 D_{\varpi}^{* 2}}}\right)
\end{aligned}
$$

the wave peak velocity $\mathrm{W}\left(\mathrm{LT}^{-1}\right)$ can be defined as:

$$
W=\frac{L}{t_{p}}
$$

The normalized wave peak velocity $W^{*}=W / L \quad\left(\mathrm{~T}^{-1}\right)$ is equal to the reciprocal of the time of peak $t_{p}$.

The hydraulic Peclet number $k$ can be defined as:

$$
k=\frac{c_{\bar{\sigma}}}{D_{\bar{\varpi}}} L \Rightarrow k=\frac{c_{\bar{\sigma}}^{*}}{D_{\bar{\sigma}}^{*}}
$$

that accounts for the relative influence of the hydraulic diffusivity and celerity. According to Rasmussen at al. (2000), the hydraulic Peclet number is a function of the slope of a semilogarithmic plot between the hydraulic head and the unsaturated hydraulic conductivity.

$k=\frac{d K / d h}{K_{a}} L \Rightarrow k=\frac{d \ln K}{d h} L$

At higher values of $k$, a perturbation of the hydraulic head gives rise to a considerable change of unsaturated hydraulic conductivity, while for lower values of $k$ a perturbation of the hydraulic head affects less unsaturated hydraulic conductivity variations. This means that lower $k$ values correspond to drier conditions characterized by higher values of water tension that do not permit a relevant increase of hydraulic conductivity when hydraulic head increases, vice versa higher $k$ values correspond to more saturated conditions in which the unsaturated hydraulic conductivity increases more rapidly when the hydraulic head increases.

This means that, under drier conditions, with increasing water tension, the hydraulic Peclet number decreases and the hydraulic diffusivity starts being more relevant with values of wave peak velocities higher than the kinematic velocity. In this case rapid wave velocities are mostly due to the diffusion of the flow wave.

With increasing saturation, the hydraulic Peclet number increases and the celerity starts increasing and equals the wave peak velocity. For higher values of hydraulic Peclet number, the flow wave velocities dominate yielding a flow wave velocity consistent with the kinematic theory (advection of the flow wave).

According to Nimmo (2010), assuming that the diffuse flux recharge reaches the water table and the rate of decline of the water table without recharge is proportional to the hydraulic head, the governing equation that controls groundwater hydraulic head fluctuation $H(\mathrm{~L})$ due to recharge flux $q(L, t)$ can be expressed as:

$$
\frac{d H}{d t}=\frac{q(t)}{S_{Y}}-\frac{H}{\tau}
$$

where $S_{y}(-)$ is the specific yield of the saturated medium and $\tau$ (T) is the recession time. Equation (20) presents an analytical solution that can be applied for discrete time intervals where for each of them $q(t)$ can be considered constant.

Thus the solution of the water table fluctuation in recursion form can be written according with Nimmo (2010) as:

$$
H_{i+1}=H_{i} \exp \left(-\frac{\Delta t}{\tau}\right)+\frac{q\left(t_{i+1}\right)}{S_{Y}} \tau\left[1-\exp \left(-\frac{\Delta t}{\tau}\right)\right]
$$

\section{RESULTS AND DISCUSSION}

The kinematic diffusion wave model has been applied to evaluate the specific flow rate that reaches the water table. The recession time, the normalized celerity and the normalized hydraulic diffusivity have been estimated for each single episodic recharge event comparing the observed and the simulated hydraulic head obtained by Equation (20).

Table 2 and Table 3 show the mm of precipitation and the rainy days per season during the period 2007-2013 respectively.

The average amount of rainfall over the whole period is 617 $\mathrm{mm}$, less than the average measured annual rainfall of 669.4 $\mathrm{mm}$. The distribution of rainfall over the four seasons is quite regular and shows homogeneous characteristics. Precipitations are spread fairly evenly throughout the year without much difference between the least rainy season (spring) and the rest of the year. The only one exception is for spring 2011 that proves to be a very dry season with only 14 rainy days and 19 $\mathrm{mm}$ of rain and spring 2008 which has a very wet behaviour (241.4 mm over 60 days).

Generally, there are more rainy days in the winter/autumn season but the amount of rainfall is less, whereas in the summer/spring season the number of rainy days is less but the amount of rainfall is higher, having the precipitations prevalently a stormy character in the latter season.

Figure 3 shows the comparison between the daily precipitation and specific flow rate that reaches the water table together with the graphs of the observed and simulated hydraulic heads for each analysed year. The model parameters have been estimated for each precipitation event, therefore the observed data were fitted starting from an initial condition corresponding to the simulated hydraulic head of the previous fitting and choosing as the period of simulation the duration of the precipitation event. 

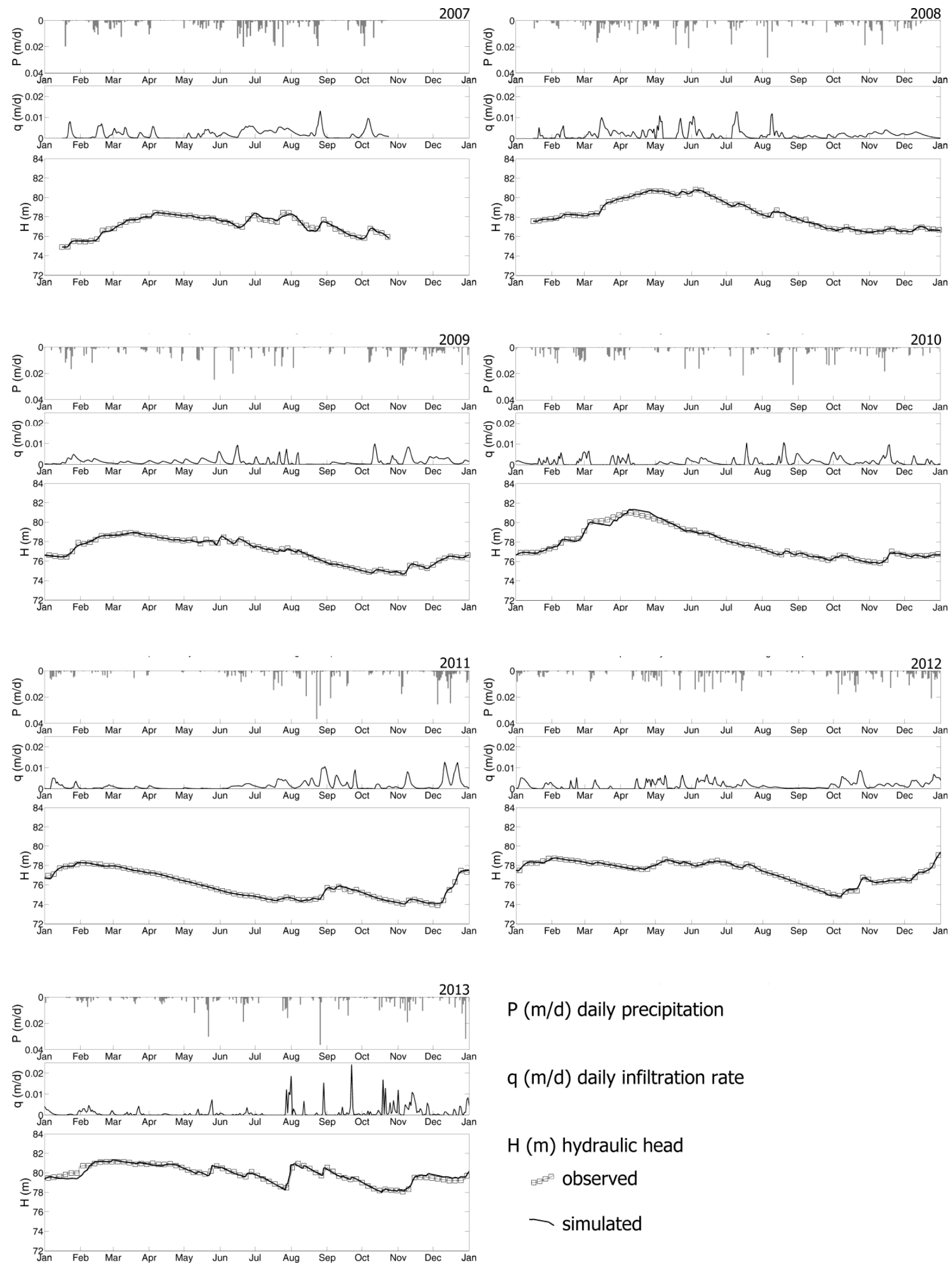

\section{$P(m / d)$ daily precipitation}

\section{$\mathrm{q}(\mathrm{m} / \mathrm{d})$ daily infiltration rate}

\section{$\mathrm{H}(\mathrm{m})$ hydraulic head

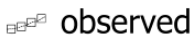 \\ simulated}

Fig. 3. Daily precipitation $P(\mathrm{~m} / \mathrm{d})$, daily specific infiltration flow rate $q(\mathrm{~m} / \mathrm{d})$ that reaches the water table and simulated and observed hydraulic heads $H(\mathrm{~m})$ for the period 2007-2013.

The kinematic diffusion model provides a satisfactory fitting both for the rising and falling periods. From an analysis of the hydraulic head data, there is the evidence of 1) a seasonal behaviour characterized by a constant increase in the water level during the late autumn and winter period (which may extend to the beginning of the spring) and 2) a recession period with a series of more episodic behaviours during the spring/summer season. The years 2007 and 2013 are characterized by high 
fluctuations of the water table during the summer season whereas 2008 and 2010 are characterized by lower water tables concentrated in autumn.

Table 4. Mean and standard deviation of recession time $\tau$ (d), normalized celerity $C_{\omega} L\left(\mathrm{~d}^{-1}\right)$ and normalized hydraulic diffusivity $D_{\omega} / L^{2}\left(\mathrm{~d}^{-1}\right)$ for the Spring/Summer and Autumn/Winter.

\begin{tabular}{lcccc}
\hline & & $\tau(\mathrm{d}) \times 10^{3}$ & $C_{\omega} / L\left(\mathrm{~d}^{-1}\right)$ & $D_{\omega} / L^{2}\left(\mathrm{~d}^{-1}\right)$ \\
\hline Spring/Summer & $\mu$ & 1.631 & 0.238 & 0.032 \\
& $\sigma$ & 1.023 & 0.094 & 0.035 \\
\hline Autumn/Winter & $\mu$ & 2.713 & 0.232 & 0.031 \\
& $\sigma$ & 1.957 & 0.103 & 0.038 \\
\hline
\end{tabular}

In Table 4 are reported the mean and standard deviation of the estimated values for the autumn/winter season (JanuaryApril, October-December) and the late spring/summer (MaySeptember). A similar behaviour for the normalized celerity and the normalized hydraulic diffusivity has been observed for the two periods, while a different behaviour is shown for the recession time.

The late autumn/winter period characterised by a seasonal behaviour has longer recession times than the summer season having an episodic behaviour, in that the slope of the falling limb during each precipitation event is higher in the latter than in the former. During the prevalence of episodic behaviours which normally happen during the recession period, the water level responds to successive heavy rainfall events after a time delay, when it may suddenly rise with a high slope afterwards (August 2008, June-July 2009, August 2011).

Figure $4 \mathrm{a}$ shows the normalized celerity vs the normalized diffusivity. Figure $4 \mathrm{~b}$ shows the residence time $t_{m}$ vs. the hydraulic Peclet number $k$. The latter is always higher than the unit with a mean value equal to 7.48 therefore kinematic phenomena dominate over diffusive ones. As celerity increases diffusivity decreases, the kinematic phenomena become dominant and therefore the residence time decreases, whereas for lower velocities diffusive phenomena become more relevant thus increasing the residence time.

For higher values of normalized celerity, the system is advection-dominated and fractures seem to act as the primary flow pathway that causes rapid water-level fluctuations. For lower celerity values, fracture matrix interaction through diffusive phenomena starts being relevant.

Figure 5 shows the relationship between the residence time and the time variance $\sigma^{2}$ directly related to the hydraulic diffusivity. For $t_{m}<3$ days $\sigma^{2}$ fluctuates around a value of $10 \mathrm{~d}^{2}$ and for $t_{m}>3$ days it starts increasing. This means that the diffusive behaviour becomes more important with increasing the residence time.

Figure 6a shows the relationship between the normalized celerity and the normalized wave peak velocity. For a value of normalized celerity higher than $0.15 \mathrm{~d}^{-1} W / L$ matches the value of $C_{\omega} / L$. For a value of $C_{\omega} L$ less than $0.15 \mathrm{~d}^{-1}, W / L$ deviates from $C_{\omega} L$ resulting higher.

Figure $6 \mathrm{~b}$ shows the relationship between the hydraulic Peclet number and the ratio between the wave peak velocity and celerity. As the hydraulic Peclet number increases the ratio between the wave peak velocity and celerity matches the unit. This means that, under drier conditions, with decreasing $k$ and increasing water tension, the hydraulic Peclet number decreases and the hydraulic diffusivity starts being more relevant. The infiltration rate goes through a few flow paths corresponding to values of wave peak velocity higher than the kinematic velocity. In this case, rapid wave velocities are mostly due to the diffu-
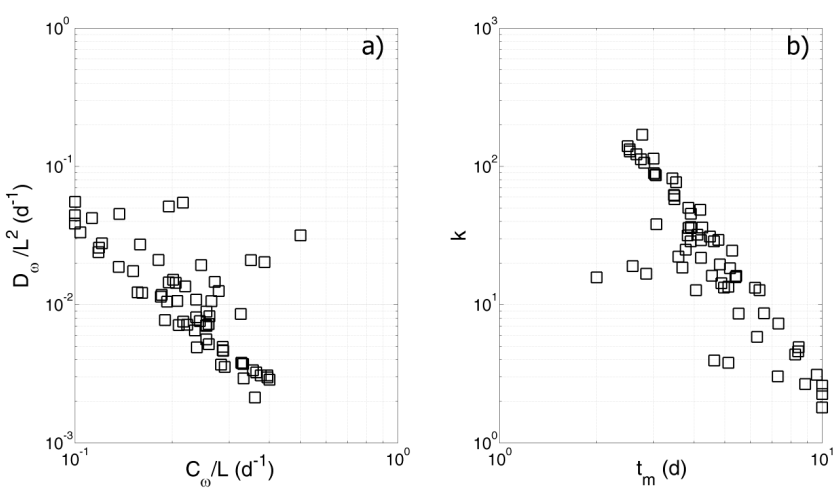

Fig. 4. a) Hydraulic diffusivity $D_{\omega} / L^{2}$ versus Normalized celerity $C_{\omega} L$; b) hydraulic Peclet number $k$ versus residence time of the unit response function.

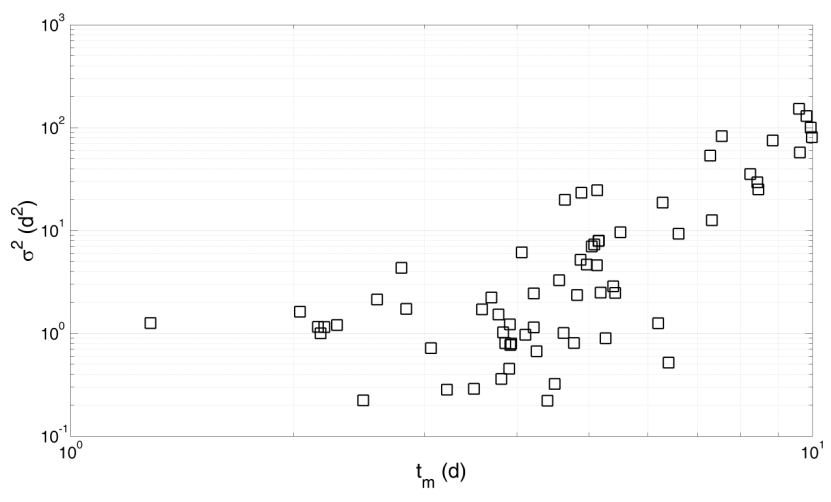

Fig. 5. Time variance $\sigma^{2}$ versus residence time $\mathrm{t}_{\mathrm{m}}$ evaluated on the unit response functions.
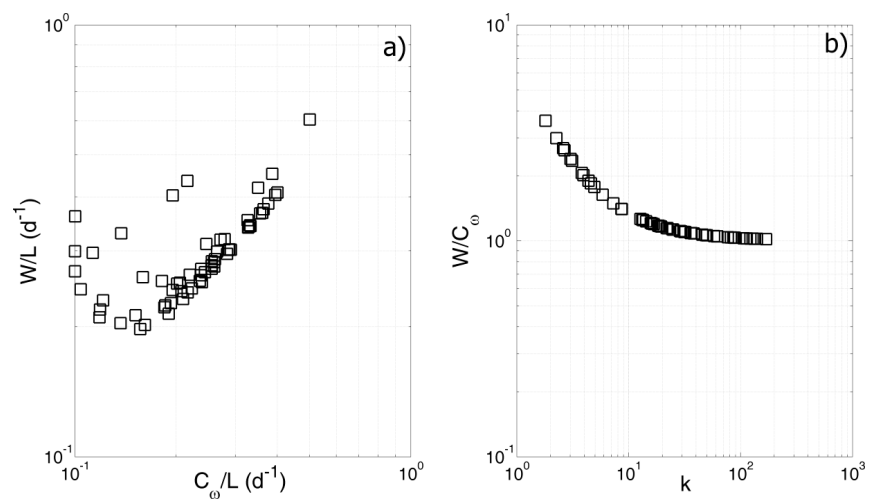

Fig. 6. a) Normalized wave peak velocity $W / L\left(\mathrm{~d}^{-1}\right)$ versus normalized celerity $C_{\omega} / L\left(\mathrm{~d}^{-1}\right) \mathrm{vs}$. b) ratio between wave peak velocity and celerity $\mathrm{W} / \mathrm{C}_{\omega}$ versus hydraulic Peclet number $\mathrm{k}$.

sion of the flow wave. With increasing saturation, the hydraulic Peclet number increases, the flow paths are all active and celerity starts increasing and equals the wave peak velocity. For higher values of hydraulic Peclet number the flow wave velocities dominate yielding a value consistent with the kinematic theory (advection of the flow wave). This is coherent with the experiments carried out by Rasmussen et al. (2000) who put forward evidence for diffusion-dominated soil water pressure waves whose velocities initially exceeded kinematic wave celerities in the more shallow depths of intact saprolite columns. Subsequently, diffusion- dominated soil water pressure wave velocities decreased with depth, conforming with kinematic wave theory. 


\section{CONCLUSIONS}

The hydrologic understanding and predicting of recharge mechanisms in chalk aquifers is essential for effective water resources managements especially in North West Europe where Chalk constitutes the most important source of fresh water.

In order to evaluate the specific flow rate that reaches the water table in the Cretaceous fractured chalk aquifer in Picardy (North of France), the kinematic diffusion theory has been applied that treats the unsaturated water flow equation as a wave equation composed of diffusive and gravitational components. This model has proved to be a valuable approach for the prediction of the unsaturated zone flux recharging the chalk aquifer in that it has provided a satisfactory fitting both where water table fluctuations in response to rainfall showed the evidence of a seasonal and an episodic behaviour.

The hydraulic Peclet number being always higher than the unit and the negative correlation between normalized celerity and hydraulic diffusivity prove that kinematic phenomena dominate over diffusive ones. For higher values of normalized celerity, the system is advection dominated. The shallow fractures form preferential flow paths and dominate the vadose zone recharge processes, as reflected in the ground water-table elevation rise. They also influence the ground water-table recession rate, reflected in the rapid water-table recession (Qin, 2014). For lower celerity values, hydraulic diffusivity becomes more important, being matrix and fracture-matrix interaction most responsible of diffusive phenomena.

The comparison between the normalized celerity and the normalized wave peak velocity puts into evidence that for a value of normalized velocity higher than $0.15 \mathrm{~d}^{-1}$ kinematic phenomena are dominant, whereas for a value of normalized velocity less than $0.15 \mathrm{~d}^{-1}$ the effect of diffusive phenomena increases, being the normalized celerity lower than the normalized wave peak velocity.

For higher values of hydraulic Peclet number (increasing saturation) the pressure wave velocities dominate yielding a flow wave velocity consistent with the kinematic theory (advection of the flow wave). With decreasing values of hydraulic Peclet number (increasing water tension), rapid wave velocities are mostly due to the diffusion of the flow wave.

This study has applied the kinematic wave theory to model infiltration in unsaturated fractured chalk. The kinematic diffusion model constitutes a reliable approach to predict water table response and recharge over many months, giving a satisfactory fitting both for rising and falling periods.

It has also proved to give an answer to the question whether unsaturated flow can be described using the theory of kinematic waves. The answer to the question depends principally on the status of soil moisture.

The movement of water through unsaturated fractured rock is due to capillary and gravity forces. The relative significance of each of them depends on the formation characteristics and again its moisture content. For example, when the upper boundary is subjected to high flux, or is suddenly saturated, flow into a dry soil is dominated by capillary potential gradients in the matrix (Singh, 1997), whereas flow may remain mostly gravity driven in fractures. At low moisture contents, capillary forces are stronger and flow is dominated by the diffusion wave, although the kinematic wave also exists. With increasing moisture content, the diffusion wave decreases and the kinematic wave begins to be more relevant. Thus, at a fixed position in soil both waves exist at a time but their relative magnitudes are governed by the moisture content at that time (Singh, 2002).
The use of a kinematic wave in this context constitutes a good simplified approach especially in cases when there is a lack of information concerning the hydraulic properties of the fractures/macropores close to saturation (Larsbo et al., 2005).

The kinematic wave approach treats unsaturated fracture flow as a non-capillary, gravity-dominated process, postulating that the moisture flux is a function of only the moisture content. Because it neglects capillary effects, the calculation of the fracture flow velocity is only approximate.

Current and future developments of the present study will be to deepen the analysis of infiltration, percolation and recharge in unsaturated fractured rock by carrying out laboratory tests. An apparatus has been set up at the hydraulic laboratory of the University of Queensland to test infiltration in synthetic fractures with varying geometrical configurations and angles of inclinations, in order to analyse the influence of aperture, roughness and slope on infiltration dynamics.

This will improve the understanding of the physical processes in the unsaturated zone in relation to the assessment of the Chalk aquifer's recharge and of its vulnerability to contamination.

Acknowledgements. The authors would like to acknowledge Dr Nicoletta Allegretti for her contribution to the paper as well as the editors and the two anonymous reviewers for their valuable comments and suggestions to improve the quality of the paper.

\section{REFERENCES}

Barhoum, S., Valdfies, D., Gufierin, R., Marlin, C., Vitale, Q., Benmamar, J., Gombert, P., 2014. Spatial heterogeneity of high-resolution Chalk groundwater geochemistry - Underground quarry at Saint Martin-le-Noeud. Journal of Hydrology, 519, Part A, 756-768.

Beven, K., 1982. On subsurface stormflow: predictions with simple kinematic theory for saturated and unsaturated flows. Water Resources Research, 18, 6, 1627-1633.

Brouyère, S., Dassargues, A., Hallet, V., 2004. Migration of contaminants through the unsaturated zone overlying the Hesbaye chalky aquifer in Belgium: a field investigation. J. Contam. Hydrol., 72, 1-4, 135-64.

Brutsaert, W., 2005. Hydrology an Introduction. Cambridge University Press, New York.

Charbeneau, R.J., 1984. Kinematic models for soil moisture and solute transport. Water Resources Research, 20, 6, 699706.

Charbeneau, R.J., Weaver, J.W., Smith, V.J., 1989. Kinematic modelling of multiphase solute transport in the vadose zone. EPA Report EPA/600/2-89/035, R.S.K. Environmental Research Laboratory, US Environmental Protection Agency, Ada, OK, $1588 \mathrm{p}$.

Crampon, N., Roux, J.C., Bracq, P., 1993. Hydrogéologie de la craie en France. Hydrogéologie, 2, 81-123.

Crank, J., 1956. Mathematics of Diffusion. Oxford University Press, New York, London.

Dahan, O., Nativ, R., Adara, E.M., Berkowitz, B., Ronen, Z., 1999. Field observation of flow in a fracture intersecting unsaturated chalk. Water Resour. Res., 35, 11, 3315-3326.

Downing, R.A., Pearson, F.J., Smith, D.B., 1979. The flow mechanism in the Chalk based on radio-isotope analyses of groundwater in the London Basin. Journal of Hydrology, 40, $1-2,67-83$.

Foster, S.S.D., 1975. The Chalk groundwater tritium anomaly a possible explanation. Journal of Hydrology, 25, 159-165. 
Germann, P., Beven, K., 1985. Kinematic wave approximation to infiltration into soils with sorbing macropores. Water Resources Research, 21, 990-996.

Germann, P., Beven, K., 1986. A distribution function approach to waterflow in soil macropores based on kinematic wave theory. Journal of Hydrology, 83, 173-183.

Ireson, A.M., Butler, A.P., 2011. Controls on preferential recharge to Chalk aquifers. J. Hydrol., 398, 109-123.

Larsbo, M., Roulier, S., Stenemo, F., Kasteel, R., Jarvis, N., 2005. An improved dual permeability model of water flow and solute transport in the vadose zone. Vadose Zone Journal, 4, 398-406.

Lighthill M.J., Whitham, G.B., 1955. On kinematic waves: 1. Flood movement in long rivers. Proceedings Royal Society London, Series A, 229, 281-316.

Nicholl, M.J., Glass, R.J., Wheatcraft, S.W., 1994. Gravitydriven infiltration instability in initially dry nonhorizontal fractures. Water Resour. Res., 30, 9, 2533-2546.

Nimmo, J.R., 2010. Theory for source-responsive and freesurface film modeling of unsaturated flow. Vadose Zone J., 9, 2, 295-306. DOI: 10.2136/vzj2009.0085.

Nimmo, J.R., Mitchell, L., 2013. Predicting vertically nonsequential wetting patterns with a source-responsive model. Vadose Zone J., 12, 4. DOI: 10.2136/vzj2013.03.0054.

Nimmo, J.R., Malek-Mohammadi, S., 2015. Quantifying water flow and retention in an unsaturated fracture-facial domain. In: Faybishenko, B., Benson, S.M., Gale, J.E. (Eds.): Fluid Dynamics in Complex Fractured-Porous Systems. American Geophysical Union, Washington, pp. 5-17. DOI: 10.1002/9781118877517.ch12.

Price, M., Low, R.G., McCann, C., 2000. Mechanisms of water storage and flow in the unsaturated zone of the Chalk aquifer. J. Hydrology, 233, 1-4, 54-71. DOI: 10.1016/S00221694(00)00222-5.

Qin, Z., 2014. An Unsaturated Zone Flux Study in a Highlyfractured Bedrock Area: Ground Water Recharge Processes at the Masser Recharge Site, East-central Pennsylvania. Master's Thesis. Paper 4512. San José State University, San José, CA. http://scholarworks.sjsu.edu/etd_theses/4512

Rasmussen, T.C., 2001. Pressure wave vs. tracer velocities through unsaturated fractured rock. In: Evans, D.D., Nicholson, T.J., Rasmussen, T.C. (Eds.): Flow and Transport through Unsaturated Fractured Rock. 2nd Ed. Geophysical Monograph 42, American Geophysical Union, Washington, DC, pp. 45-52.

Rasmussen, T.C., Baldwin Jr, R.H., Dowd, J.F., Williams, A.G., 2000. Tracer vs. pressure wave velocities through unsaturated saprolite. Soil Science Society of America Journal, 64, 1, 75-85.

Reeves, M.J., 1979. Recharge and pollution of the English Chalk: some possible mechanisms. Eng. Geol., 14, 231-240.

Roux, J.C., Tirat, M., 1967. Carte de la surface piezometrique de la nappe de la craie en Picardie. Bureau de Recherches Geologiques et Minieres, Service Geologique Regional Picardie Normandie.

Scheurer, O., 2000. Atlas Agriculture environnement de 1'Oise Relations spatiales entre sensibilité des sols et activité agricole.
Institut Supérieur d'Agriculture de Beauvais, Beauvais, 41 p. http://www.sols-et-territoires.org/bibliographie/applicationsthematiques/?L $=0$

Šimůnek, J., Jarvis, N.J., van Genuchten, M.T., Gärdenäs, A., 2003. Review and comparison of models for describing nonequilibrium and preferential flow and transport in the vadose zone. Journal of Hydrology, 272, 14-35.

Singh, V.P., 1997. Kinematic Wave Modeling in Water Resources, Environmental Hydrology. John Wiley, New York.

Singh, V.P., 2002. Is hydrology kinematic? Hydrological Processes, 16, 667-716. DOI: 10.1002/hyp.306.

Sisson, F.B., Ferguson, A.H., van Genuchten, M.T., 1980. Simple method for predicting drainage from field plots. Soil Sci. Soc. Am. J., 44, 1147-1152.

Smith, R.E., 1983. Approximate soil water movement by kinematic characteristics. Soil Sci. Soc. Am. J., 47, 3-8.

Su, G.W., Geller, J.T., Pruess, K., Wen, F., 1999. Experimental studies of water seepage and intermittent flow in unsaturated, rough-walled fractures. Water Resour. Res., 35, 4, 10191037.

Tokunaga, T.K., Wan, J., 1997. Water film flow along fracture surfaces of porous rock. Water Resour. Res., 33, 6, 12871295.

Valdes, D., Dupont, J., Laignel, B., Slimani, S., Delbart, C., 2014. Infiltration processes in karstic chalk investigated through a spatial analysis of the geochemical properties of the groundwater: The effect of the superficial layer of claywith-flints. Journal of Hydrology, 519, 23-33.

Van den Daele, G.F.A., Barker, J.A., Connell, L.D., Atkinson, T.C., Darling, W.G., Cooper, J.D., 2007. Unsaturated flow and solute transport through the Chalk: Tracer test and dual permeability modelling. J. Hydrol., 342, 1-2, 157-172. DOI: 10.1016/j.jhydrol.2007.05.021.

White, R.E., 1985. The influence of macropores on the transport of dissolved and suspended matter through soil. Adv. Soil Sci., 3, 95-120.

Yamada, T., Kobayashi, M., 1988. Kinematic wave characteristics and new equations of unsaturated infiltration. Journal of Hydrology, 102, 257-266.

Yang, T., 2000. Some recent results on compressible flow with vacuum. Taiwanese Journal of Mathematics, 4, 1, 33-44.

Yang, Y., Endreny, T.A., 2013. Watershed hydrograph model based on surface flow diffusion. Water Resources Research, 49, 507-516.

Zghibi, A., Chenini, I., Zouhri, L., Merzougui, A., Tarhouni, J., 2015. Modelling of tracer movement with advectiondispersion scheme at the LaSalle Beauvais experimental site, Beauvais, France. J. Hydrogeol. Hydrol. Eng., 4, 3. http://dx.doi.org/10.4172/2325-9647.1000126

Zouhri, L., Lutz, P., 2010. A comparison of peak and plate electrodes in electrical resistivity tomography: application to the chalky groundwater of the Beauvais aquifer (northern part of the Paris basin, France). Hydrol. Process., 24, 30403052 .

Received 26 July 2016 Accepted 24 May 2017 